\title{
The burden of childhood mental disorders
}

\author{
Guilherme V. Polanczyk
}

Published online: 21 February 2013

(C) Springer-Verlag Berlin Heidelberg 2013

The Global Burden of Disease Study 2010 (GBD 2010) was launched in December 2012 and a special issue of The Lancet was published, dedicated to reporting the results [1]. GBD 2010 is a systematic and comprehensive assessment of data on 291 diseases and injuries and 235 causes of death for 20 age groups, both sexes, and for 187 countries, producing estimates of mortality and of non-fatal health outcomes [1]. GBD 2010 assessed significantly more health conditions than the previous version of the study and for the first time included attention-deficit/hyperactivity disorder, conduct disorder, and autism. This is an important advance to our field because the objective estimates of the burden of childhood mental disorders contextualize these conditions among all other medical conditions and support governmental and societal actions. Results show that there is an increasing burden of non-communicable diseases and mental illnesses and substance use disorders are among the main causes of disability [1].

Mental and behavioral disorders caused approximately 185 million disability-adjusted life years (DALYs) accounting for $7.4 \%$ of DALYs globally [2]. Pervasive development disorders caused approximately 7.5 million, conduct disorder caused approximately 6 million and ADHD caused approximately half a million DALYs [2]. Mental and behavioral disorders caused approximately 177

G. V. Polanczyk $(\bowtie)$

Department of Psychiatry, Medical School and Research Support Center on Neurodevelopment and Mental Health,

University of São Paulo, Rua Dr. Ovídio Pires de Campos 785,

São Paulo, SP 05403-010, Brazil

e-mail: gvp@usp.br

G. V. Polanczyk

National Institute of Developmental Psychiatry for Children and Adolescents (INCT-CNPq), Rua Dr. Ovídio Pires de Campos 785, São Paulo, SP 05403-010, Brazil million years lived with disability (YLDs) accounting for $22.7 \%$ of YLDs globally [3]. Pervasive development disorders caused approximately 7.6 million, conduct disorder caused approximately 5.7 million and ADHD caused approximately 400,000 YLDs [3]. The process of estimating DALYs and YLDs is complex and depends on the available literature, which imposed limitations in the process possible resulting in underestimates of the burden of childhood mental disorders.

Due to the limitations imposed by the literature, mortality was not calculated for childhood mental disorders [4]. There is no doubt that mental disorders in childhood are associated with premature death, as Halfon's et al. [5] study demonstrated by examining studies on suicidality in youth bipolar disorder and summarizing the risk and protective factors detected by individual studies. Youth bipolar disorder is strongly associated with suicidal behavior, as indicated by several studies and more recently by the National Comorbidity Survey Replication Adolescent Supplement in the general population of the US [6]. A number of clinical characteristics that further increase the risk for suicidal behavior were identified, such as an acute episode, psychotic symptoms, and comorbid conditions, in conjunction with psychological characteristics, family and social factors. Of note, dialectical behavior therapy was found to protect against suicidality by improving emotional regulation and depressive symptoms. Additionally, authors were not able to find studies assessing the effect of lithium on suicidal risk. As this medication was found to decrease the risk of suicide in adults with bipolar disorder, it would be highly relevant to test if this effect also applies to youths.

Another limitation imposed to the GBD 2010 study is the fact that childhood disorders are associated to complex chains of risk factors as causes and consequences, 
challenging the process of attributing health conditions and death to a specific cause. Children who live or work on the streets are an example. This is a major social problem in developing countries, associated with poverty, migration, and population growth. Children go to the streets due to behavioral conditions, economic needs, but also as a way to escape from family violence. Once on the streets, they become exposed to urban violence and to opportunities for substance use, crimes and prostitution. In addition, due to the nature of their situation, it is difficult to keep them engaged in services and researchers face several difficulties in reaching them. Only in the city of São Paulo, Brazil, it is estimated that approximately 2,000 children live or work on the streets (http://www.fipe.org.br). Thus, this is an extremely high-risk group of children to whom efforts should be urgently directed. In this respect, the study by Maciel et al. [7] conducted in São Paulo that appears in this issue is very welcome. Authors showed that $62 \%$ of the children screened positive for psychopathology and $50 \%$ of them received a psychiatric diagnosis based on the DSM-IV according to primary caregiver, which may be substantially under-reported. Thirty-three percent of the sample reported emotional and physical abuse and $18 \%$ reported some sort of sexual abuse. Half of the sample was exposed to neglect. The rates are expressive, especially if we consider that this is young sample, with a mean age of 10 years, before the peak incidence of several disorders such as mood and substance use. Children's psychopathology was predicted by the caregiver's psychopathology, the absence of a partner in the house, and physical violence as a disciplinary method. These results indicate that a comprehensive program of intervention is necessary, with different outcomes targeting not only the child but also their families.

Comorbidity among disorders is also challenging for the process of attributing to a single condition adverse outcomes as in the GBD study. Gren-Landell et al.'s [8] study is an example of such complexes relationships that exist between mental disorders. The authors explored the relationship between social anxiety disorder (SAD) and posttraumatic stress disorder (PTSD) in a school-based sample of 5,960 youth aged 16-20 years. This relationship has been understudied in children, although it has been reported in adults that individuals with SAD reacted with symptoms like re-experiencing, avoidance and hyperarousal in relation to memories of socially stressful events. Also, studies report that socially phobic individuals perceive social events as more distressing than life-threatening events. Self-reported SAD and PTSD symptoms were found in 10.2 and $14.8 \%$ of the sample, respectively. Individuals with self-reported SAD reported significantly higher rates of victimizing experiences as well as higher levels of post-traumatic stress symptoms. This study underscores a poorly explored relationship between the two disorders that once combined are expected to have an important impact in terms of disability.

Finally, childhood mental disorders can have a negative influence on the emotional development of the individual. Although these disorders are frequently a cause of great suffering, the emotional influences are rarely adequate captured by longitudinal studies. Hoekstra's et al. [9] followed-up children with tics who participated in a large community cohort from Norway and studied its emotional impact over time. Children with tics had significantly higher parent- and teacher-rated symptoms as assessed by the Strength and Difficulties Questionnaire when they were 7-9 and 11-13 in comparison to children without tics. In addition, children with tics were matched to children without tics with comparable level of symptoms and the trajectory of symptoms was assessed. Peer and emotional problems in children with tics increased over the 4-year time period, in contrast to conduct and hyperactivity-inattention problems that decreased at a similar level as the control group. Results were similar irrespective of the informant.

Some of the mentioned limitations are intrinsic to mental disorders and can hardly be overcome. Other limitations are imposed by the researchers and can be modified. For example, there is still more emphasis in our studies on symptoms and diagnosis as outcomes, as opposed to health-related conditions [10], which does not express adequately the impact of mental disorders and make the comparison to other conditions very difficult. We should bear in mind that the task of demonstrating the burden of childhood mental disorders is ours and the questions we raise, the design, methods and outcomes we choose for our studies can make a significance difference on that.

\section{References}

1. Horton R (2012) GBD 2010: understanding disease, injury, and risk. Lancet 380(9859):2053-2054

2. Murray CJL, Vos T, Lozano R, Naghavi M, Flaxman AD, Michaud C et al (2012) Disability-adjusted life years (DALYs) for 291 diseases and injuries in 21 regions, 1990-2010: a systematic analysis for the Global Burden of Disease Study 2010. Lancet 380(9859):2197-2223

3. Vos T, Flaxman AD, Naghavi M, Lozano R, Michaud C, Ezzati $M$ et al (2012) Years lived with disability (YLDs) for 1160 sequelae of 289 diseases and injuries 1990-2010: a systematic analysis for the Global Burden of Disease Study 2010. Lancet 380(9859):2163-2196

4. Lozano R, Naghavi M, Foreman K, Lim S, Shibuya K, Aboyans $\mathrm{V}$ et al (2012) Global and regional mortality from 235 causes of death for 20 age groups in 1990 and 2010: a systematic analysis for the Global Burden of Disease Study 2010. Lancet 380(9859): 2095-2128 
5. Halfon N, Labelle R, Cohen D, Guilé JM, Breton JJ (2013) Juvenile bipolar disorder and suicidality: a review of the last ten years of literature. Eur Child Adolesc Psychiatry. doi:10.1007/ s00787-012-0328-z

6. Nock MK, Green JG, Hwang I, McLaughlin KA, Sampson NA, Zaslavsky AM, Kessler RC (2013) Prevalence, correlates, and treatment of lifetime suicidal behavior among adolescents: results from the national comorbidity survey replication adolescent supplement. JAMA Psychiatry 9:1-11

7. Maciel MR, Mello AF, Fossaluza V, Nobrega LP, Cividanes GC, Mari JJ, Mello MF (2013) Children working on the streets in Brazil: predictors of mental health problems. Eur Child Adolesc Psychiatry. doi:10.1007/s00787-012-0335-0
8. Gren-Landell M, Aho N, Carlsson E, Jones A, Svedin CG (2013) Posttraumatic stress symptoms and mental health services utilization in adolescents with social anxiety disorder and experiences of victimization. Eur Child Adolesc Psychiatry. doi:10.1007/ s00787-012-0336-z

9. Hoekstra PJ, Lundervold AJ, Lie SA, Gillberg C, Plessen KJ (2013) Emotional development in children with tics: a longitudinal population-based study. Eur Child Adolesc Psychiatry. doi: 10.1007/s00787-012-0337-y

10. Hoagwood KE, Jensen PS, Acri MC, Olin SS, Lewandowski RE, Herman RJ (2012) Outcome domains in child mental health research since 1996: have they changed and why does it matter? J Am Acad Child Adolesc Psychiatry 51(12):1241-1260 